\title{
Negative refraction and superlens behavior in a two-dimensional photonic crystal
}

\author{
R. Moussa, ${ }^{1}$ S. Foteinopoulou, ${ }^{1}$ Lei Zhang,,${ }^{1,2}$ G. Tuttle, ${ }^{2}$ K. Guven, ${ }^{3}$ E. Ozbay, ${ }^{3}$ and C. M. Soukoulis ${ }^{1}$ \\ ${ }^{1}$ Ames Laboratory-USDOE and Department of Physics and Astronomy, Iowa State University, Ames, Iowa 50011, USA \\ ${ }^{2}$ Department of Electrical and Computer Engineering and Microelectronics Research Center, Iowa State University, \\ Ames, Iowa 50011, USA \\ ${ }^{3}$ Department of Physics, Bilkent University, Bilkent, 06533 Ankara, Turkey
}

(Received 27 September 2004; published 4 February 2005)

\begin{abstract}
We experimentally and theoretically studied a left-handed structure based on a photonic crystal (PC) with a negative refractive index. The structure consists of triangular array of rectangular dielectric bars with dielectric constant 9.61. Experimental and theoretical results demonstrate the negative refraction and the superlensing phenomena in the microwave regime. The results show high transmission for our structure for a wide range of incident angles. Furthermore, surface termination within a specific cut of the structure excite surface waves at the interface between air and PC and allow the reconstruction of evanescent waves for a better focus and better transmission. The normalized average field intensity calculated in both the source and image planes shows almost the same full width at half maximum for the source and the focused beam.
\end{abstract}

DOI: 10.1103/PhysRevB.71.085106

PACS number(s): 42.25.-p, 78.20.Ci, 41.20.Jb

Left-handed-materials (LHM) are materials with simultaneously negative dielectric permittivity $\varepsilon(r)$ and negative magnetic permeability $\mu(r)$. The phase velocity of the light wave propagating inside this medium is pointed in the opposite direction of the energy flow. Thus, the Poynting vector and wave vector are antiparallel, consequently, the light is refracted negatively. The existence and impact of such materials was pointed out by Veselago. ${ }^{1}$ Years later, several theoretical and experimental groups investigated the LHM. ${ }^{2-5}$ For LHM based on photonic crystals (PCs), Notomi studied light propagation in a strongly modulated two-dimensional (2D) PC. ${ }^{6}$ Luo et al. have studied subwavelength imagining in PCs. ${ }^{7}$ Cubukcu et al. demonstrated experimentally singlebeam negative refraction and superlensing in the valence band of 2D PCs operating in the microwave regime. ${ }^{8}$ Foteinopoulou et al. emphasized the time evolution of an EM wave as it hits the interface between a right-handed (RH) and a LH material interface. ${ }^{9}$ Pendry suggested that a slab of lossless LHM with both $\varepsilon(r)$ and $\mu(r)$ equal to -1 should behave as a perfect lens, ${ }^{10}$ i.e, the small details as well as the larger ones could be reproduced by such a lens. The reconstruction of the evanescent wave components is the key to such perfect focusing. It is shown in another study ${ }^{11}$ how the evanescent waves get amplified upon reaching the interface between a RH and LH medium and consequently how they participate in improving the quality of the image. Homogeneous metamaterials and PCs were used to demonstrate the effect. The main challenge is to find a structure with $n=-1$ for which the matching condition are verified with no reflections. The candidate structure should have three characteristics. First, it should exhibit almost isotropic equal frequency surfaces (EFS) in a band region with $\mathbf{v}_{g} \cdot \mathbf{k}<0$, with $\mathbf{k}$ being the wave vector in the first Brillouin zone (BZ) and $\mathbf{v}_{g}$ is the group velocity. The latter implies $\mathbf{S} \cdot \mathbf{k}<0,{ }^{12}$ and thus $\mathrm{LH}$ behavior. ${ }^{5}$ Second, it should guarantee the absence of any higher order Bragg reflections for any angle of incidence. Finally it should guarantee single beam propagation.

In this paper we propose such an almost ideal structure based on a PC. The proposed structure meets all the required conditions and has a high transmission at the desired frequency as well as a refractive index very close to $n=-1$. As it will be discussed below, the refractive index ranges from -0.97 to -1.22 because of the anisotropy. Additionally, with an appropriate cut and a specific termination of the surface, the proposed structure focuses the image in a better way by exciting the surface waves and enabling them to contribute toward the quality of the image. Furthermore, for different incident angles, the cut structure demonstrates a high transmission as well as a maximum coupling with the surface modes at the interfaces. The proposed structure consists of a triangular array of dielectric bars with a dielectric constant $\varepsilon=9.61$ in air. The dimensions of each bar in the $x$ and $y$ directions are, respectively, $r_{x}=0.40 a$ and $r_{y}=0.80 a$ where $a=1.5875 \mathrm{~cm}$ is the center to center separation between bars. The length of each bar is $l=45.72 \mathrm{~cm}$. Figure 1 shows the structure with more details. Only the TM modes are considered here (the E field is parallel to the rods).

The plane wave method is used to compute the photonic band structure as well as the EFS. Some of the theoretical results are obtained using the finite difference time domain (FDTD) method. This method shows a time and space evolution of the emitted EM waves. More details of this algorithm can be found in Ref. 13. In all the FDTD simulations we report, Liao boundary conditions ${ }^{14}$ are used. Depending on the desired conditions for the case under study, the source emits a monochromatic TM- (E-) polarization of desired dimensionless frequency. A Gaussian source (Gaussian in space and "almost" monochromatic) is placed outside the structure to check the negative refraction. However, a point source also excites the surface waves at the interface between RH and LH material. The photonic band structure as well as the EFS in $\mathbf{k}$ space are shown in Fig. 2. The quantity $(\mathrm{fa} / \mathrm{c})$ is the dimensionless frequency where $c$ is the velocity of light and $a$ is the lattice constant. A region in the second band, extending from 0.313 to 0.41 , has $\mathbf{v}_{g} \cdot \mathbf{k}<0$ and is of particular interest. To study the system and to compute the refractive index, the EFS are plotted for different frequen- 


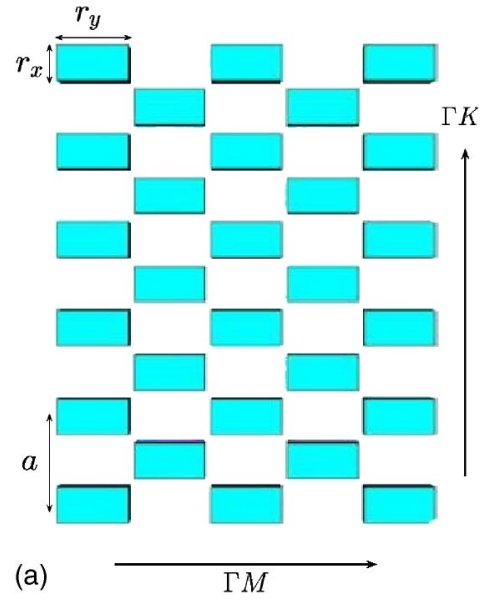

(b)

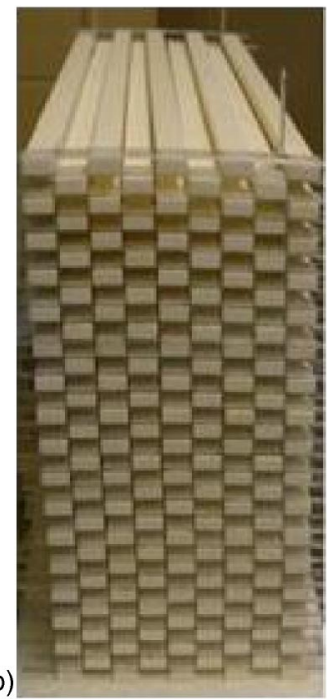

FIG. 1. (Color online) (a) A triangular array of dielectric bars in air with $r_{x}=0.40 a$ and $r_{y}=0.80 a$, where $a=1.5875 \mathrm{~cm}$ is the lattice constant. (b) A picture of the structure with 33 layers in the lateral direction and nine layers in the propagation direction.

cies. Note that the EFS consist of the allowed propagation modes for a specific frequency and in our case, they have closed concavelike form. Within the second band the shape of the EFS tends to became circular for higher frequencies and reproduces the symmetry of the system for lower frequencies. It is important to notice that in contrary with the triangular lattice with circular rods for which the hexagonal symmetry is conserved on the lattice as well as on the scatterers, this structure with rectangular rods loses its hexagonal symmetry for the scatterers when the wave is traveling along a specific direction. Thus the breaking of the symmetry gives some anisotropy in the structure. Thus, the EFS are not totally isotropic because of the breaking of the symmetry. Therefore, the computed phase refractive index ${ }^{5,6}$ of our structure using the EFS plot is somehow affected by the anisotropy. It is not exactly equal to -1 . In fact, for different incident angles it ranges from -0.97 to -1.22 .

Transmission measurements are performed to verify and test the negative refraction and superlensing. The experimental setup consists of an HP 8510C network analyzer, a waveguide horn antenna as the transmitter, and a monopole antenna as the receiver. The PC used for the negative refraction experiment has nine layers in the propagation direction $(\Gamma M)$ and 33 layers in the lateral direction $(\Gamma K)$. The interfaces are along the $\Gamma K$ direction. In all the experimental and theoretical results, the electric field is kept parallel to the bars. The operational frequency that leads to a structure of approximatively $n=-1$ is $6.5 \mathrm{GHz}$ which correspond to a dimensionless frequency of $f a / c=0.345$. For the negative refraction the horn antenna is oriented such that it makes an angle $\theta$ with the normal to the $\Gamma K$ interface.

To examine the negative refraction, we first measure the transmission along the first interface in the $\Gamma K$ direction without the PC. We repeat the measurement but this time with the PC and calculate the transmission in the vicinity of the second interface. The results are plotted in Fig. 3. It is
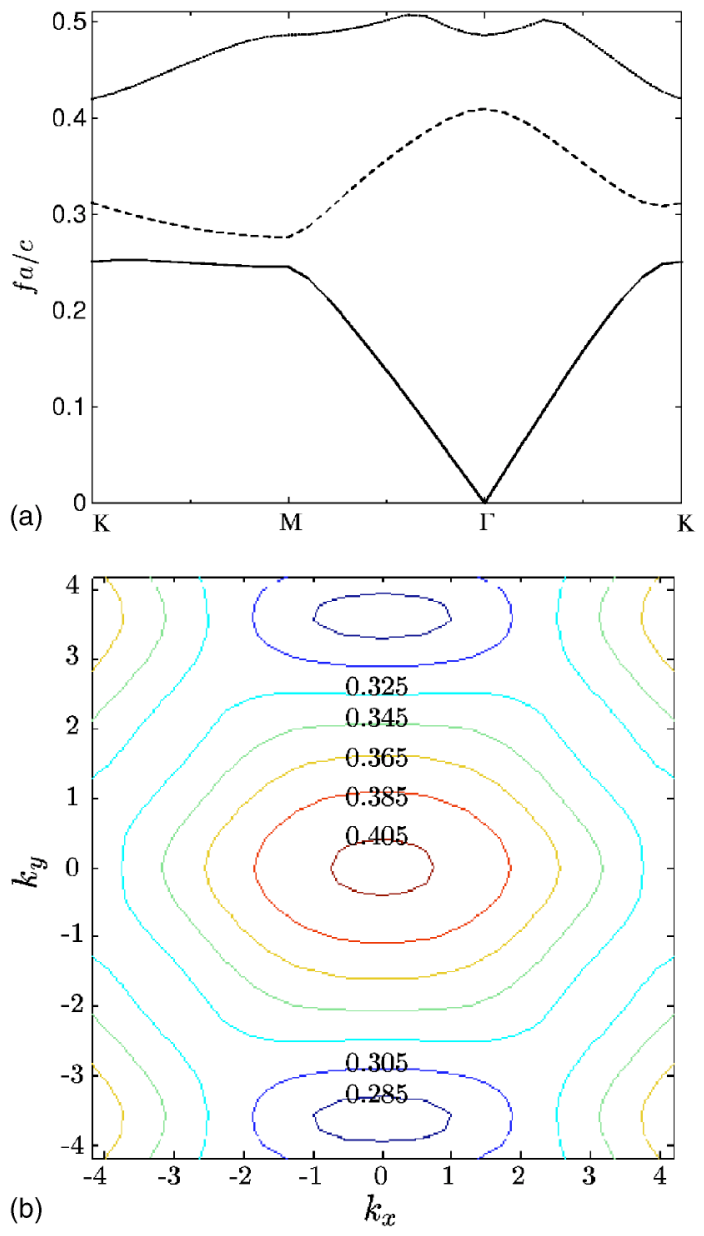

FIG. 2. (Color online) (a) The photonic band structure for the TM polarization. (b) The EFS with different dimensionless frequencies shown on the corresponding curves.

shown clearly how the center of the outgoing Gaussian beam is shifted toward the left-handed side of the center of the incident beam. This is a clear indication of the negative refraction occurring inside the structure. To get a further insight about the negative or positive shift, Fig. 4 explains in detail in which cases we have positive or negative refraction.

It is important to notice that experimentally we did observe the negative refraction for different angles. This confirms that the negative refraction is coming from the almost circular EFS, 5,7 when $\mathbf{v}_{g} \cdot \mathbf{k}<0$. For a particular angle of $\theta$ $=30(\mathrm{deg})$, the lateral shift was $3.2 \mathrm{~cm}$ which correspond to a negative angle inside the LH PC of -15 (deg). We also have done experiments for different incident angles, and always found negative refraction. In particular for incident angles of 45 and $60(\mathrm{deg})$, the refracted angles are -20 and $-30(\mathrm{deg})$, respectively.

Further insight about the field distribution inside this structure can be gained by observing the E field inside a LH PCs slab plotted in Fig. 5 after 77.33T, where $T=2 \pi / \omega$ is the period of the incident waves. The structure length along the lateral direction is about $13.8 \lambda$ and about $2.98 \lambda$ in the propagation direction. A Gaussian beam source with $\lambda=46 \mathrm{~mm}$ is placed outside the PC making an angle $\theta=30(\mathrm{deg})$ with respect to the interface which lies in the $\Gamma K$ direction. The 


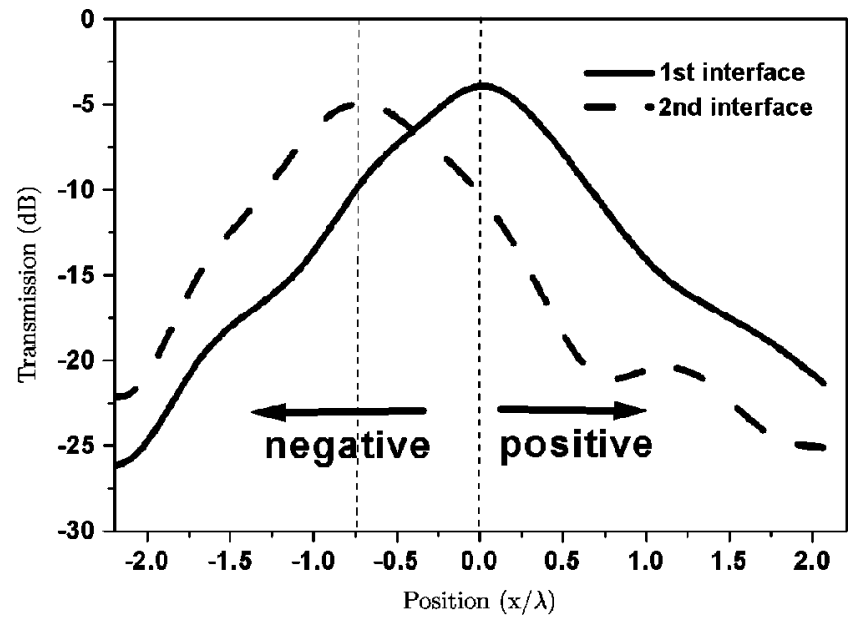

FIG. 3. The transmission at the first (solid line) and second interface (dashed line) of the PC vs the lateral position. Arrows indicate the refracted beam's direction depending on positive or negative shift.

FDTD simulation is performed by discretizing the real space into a fine rectangular grid of $(a / 54$ and $a / 92$ for the $x$ and $y$ axes, respectively). A time step of $\delta t=24.90 \mathrm{ps}$ is used. The distribution of the field inside the PC shows how the beam is negatively refracted inside the PC and refracted in parallel way to the incident beam outside the PC. The theoretical result is in a good agreement with the experiment since it gives a negative refracted angle of about -15 (deg). Theoretical agreement also exists for the other incident angles.

An important advantage of this structure is its property to allow single beam propagation. The structure was designed in such a manner that only one beam propagation is allowed eliminating the undesired Bragg waves inside the PC. ${ }^{5}$ One of the interesting features of this structure compared to the other LHM based on proposed PCs is its high transmission. Figure 6 displays the transmission versus the dimensionless frequency. The experimental curve shows the high transmission of the structure over a wide range of frequencies including the operational one. The arrows in this figure show the theoretical indication of the gaps. As predicted by the band structure, the experimental curve shows two gaps in the $\Gamma M$ direction one between 0.25 and 0.276 and the other one starting at 0.41 . Thus, the agreement between the band structure and the experimental curve is very good.

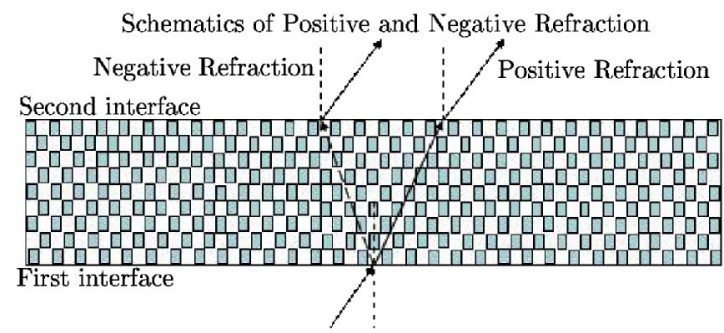

FIG. 4. (Color online) Schematics of positive and negative refraction. For negative refracted beam, the center is shifted to the left-hand side of the incident beam and for the positive refracted beam, the center is shifted to the right-hand side of the incident beam.

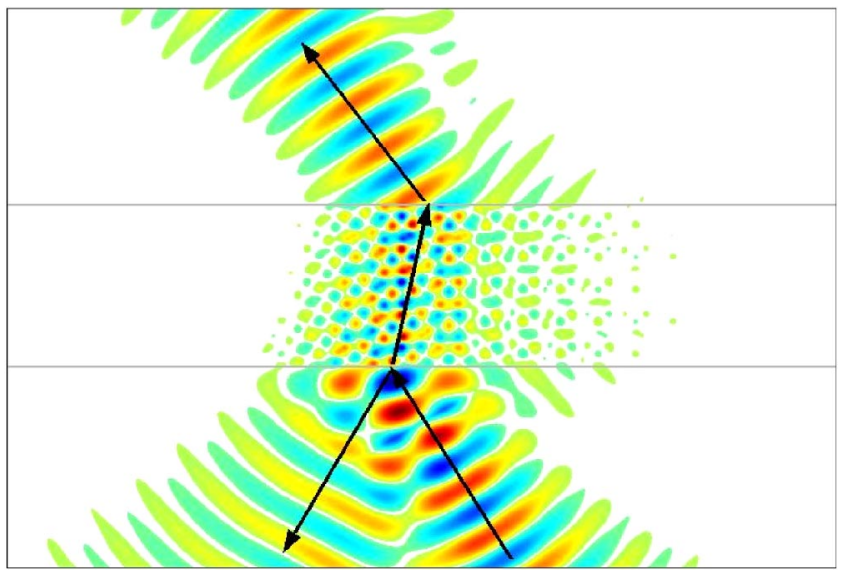

FIG. 5. (Color online) The E field of a Gaussian beam propagating along $30(\mathrm{deg})$ direction after $77.33 T$. The structure is $13.8 \lambda$ in the lateral direction and $2.98 \lambda$ in the propagation direction.

In order to get an idea about what is happening inside the structure we examine the transmission in both directions. Figure 7 shows the transmission in the $\Gamma M$ and $\Gamma K$ direction versus the frequency. This structure at the dimensionless operational frequency $(0.345)$ has a good transmission in the $\Gamma M$ direction and a weak transmission in the $\Gamma K$ direction. A weak transmission in the $\Gamma K$ direction enhance the transmission in the propagation direction which will be a critical issue for the superlensing phenomenon. It was argued ${ }^{15,16}$ that the focusing seen at the edge of the first band in a square lattice ${ }^{7}$ was not due to negative refraction but to anisotropic propagation resulting to the funneling effect. This is not the case in our structure. As we will show later, the focusing and the superlensing in our structure relies on negative refraction and on coupling to the surface states.

In order to investigate the superlensing phenomenon, we experimentally measure the intensity of the focus for the same structure used in the transmission measurements (33 layers in the lateral direction and 9 layers in the propagation direction). Two probes are used. The first one is placed at a

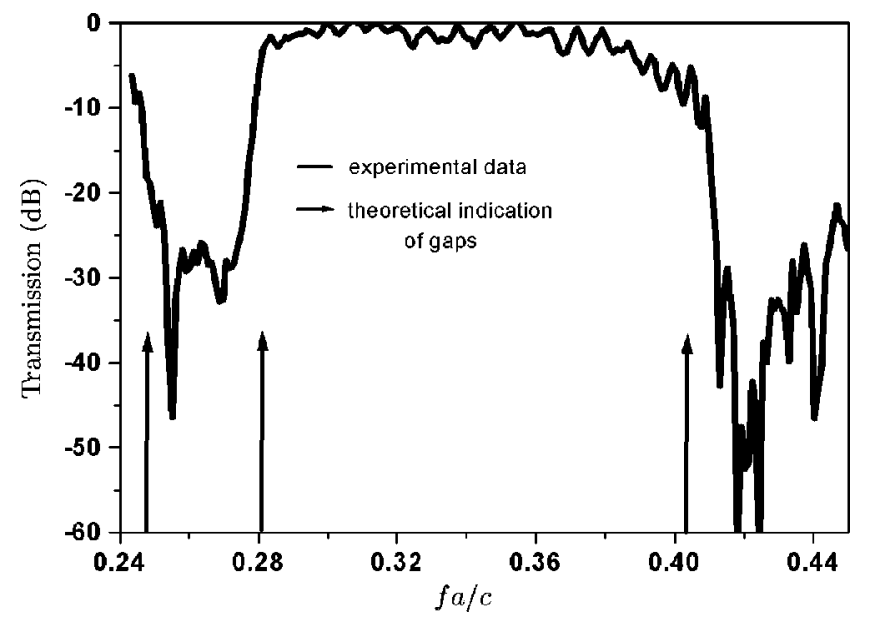

FIG. 6. The transmission along the $\Gamma M$ direction vs the dimensionless frequency. The solid arrows indicate the theoretical gaps as calculated from the band structure. 


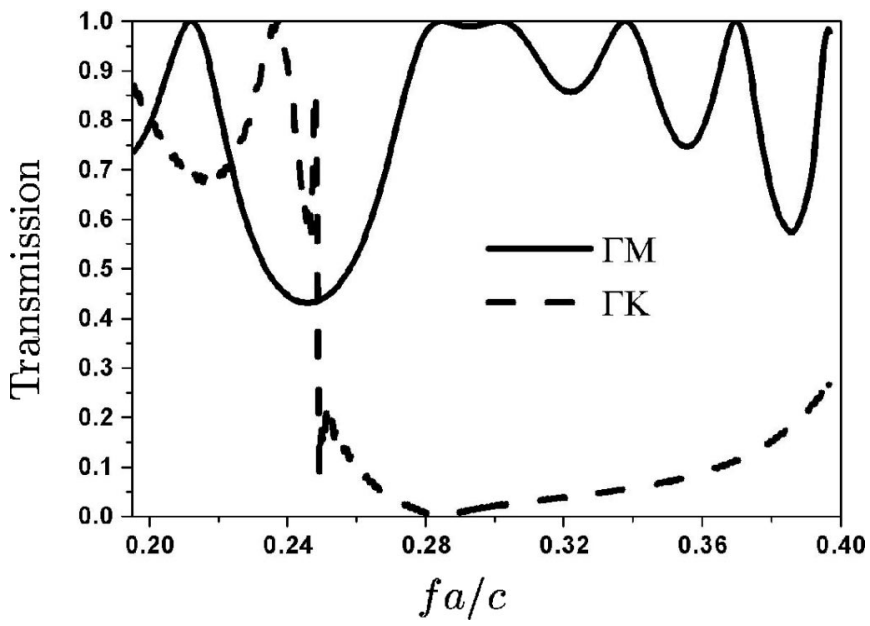

FIG. 7. The transmission vs the frequency for both the $\Gamma M$ and $\Gamma K$ direction.

distance of $0.2 \lambda$ from the first interface of the PC and the second one at the same distance from the second interface. We first measure the transmission with and without the PC. Figure 8 shows the two resulting curves. This figure demonstrates the high intensity of the focus and that the transmission is enhanced by more than $20 \mathrm{~dB}$ for the setup with the PC compared to the one without the PC. The solid curve also shows a high transmission at the edge of the structure at $x / \lambda \simeq 1.75$. This might be due to the limited lateral length of the structure and to the support station which might give some reflection at the edge.

Theoretically, we investigate the superlensing within a slab of PC with a dimension of $20.7 \lambda$ in the lateral direction and $1.20 \lambda$ in the propagation direction. A point source with a dimensionless frequency 0.345 placed at a distance $0.15 \lambda$ from the first interface of the PC is used. A time step of $\delta t$ $=26.5 \mathrm{ps}$ and a fine rectangular discretization mesh of $a / 40$ and $a / 92$ in the $x$ and $y$ direction are used. Figures 9(a) and 9(b) show the snapshots of the E field after $68.49 T$ for the

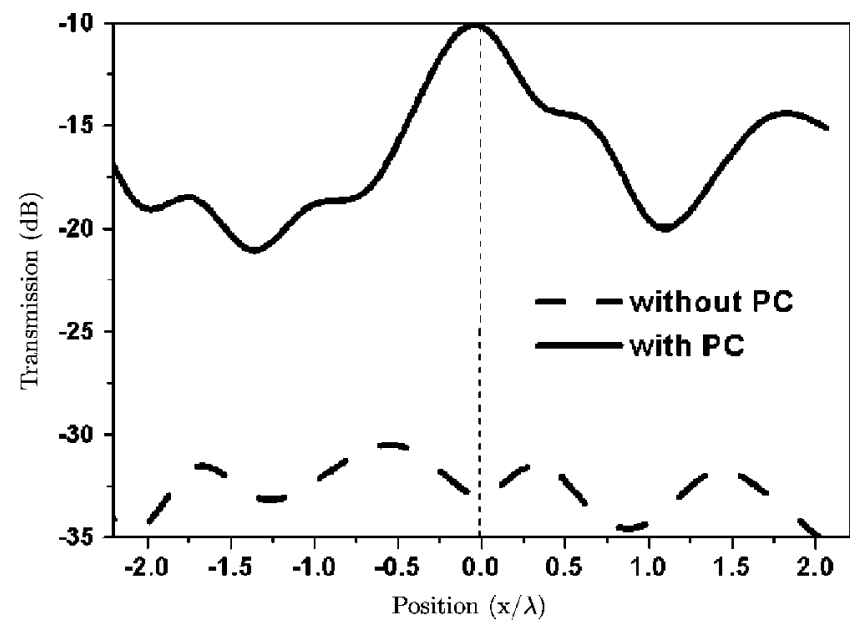

FIG. 8. The transmission vs the lateral position of a probe placed at a distance $0.2 \lambda$ from the first interface without the PC (dashed curve) and with the PC (solid curve) at $6.5 \mathrm{GHz}$ or a dimensionless frequency $(\mathrm{fa} / \mathrm{c})=0.345$.
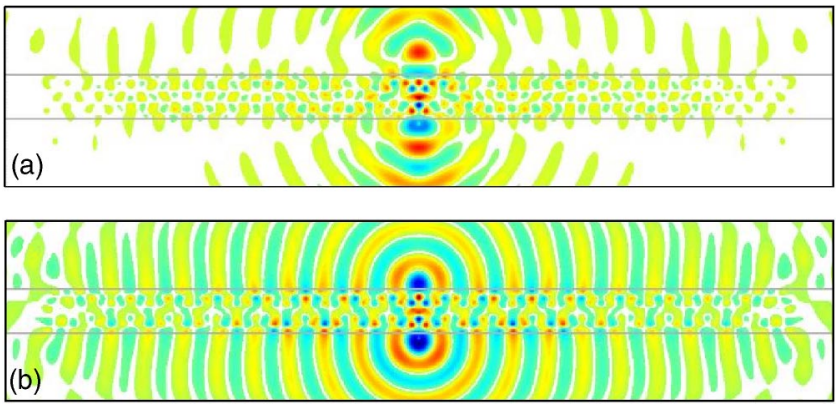

FIG. 9. (Color online) Snapshots of the E field after 68.49T of a point source located at $0.15 \lambda$ and its image through a PC slab of $20.7 \lambda$ in the lateral direction and $1.20 \lambda$ in the propagation direction. (a) shows the complete structure and (b) shows the structure with a surface cut of $0.10 a$.

complete structure and the one with $0.10 a$ termination. By the termination or cut $0.10 a$ we mean that we cut $0.10 a$ from the first and last rows of the bars. Notice that in order to excite the surface waves at the interface, we studied different terminations of the surface. ${ }^{17,18}$ Among the different cuts, one surface termination succeeds in exciting the surface waves as shown in Fig. 9(b). In this case the cut was about $0.10 a$. For both figures [Figs. 9(a) and 9(b)] a focus is obtained. However, a better image with a higher intensity is obtained when surface waves are involved. The surface waves in Fig. 9(b) are clearly shown as propagating along both the $\Gamma K$ interfaces. As shown in Fig. 9(b), the image is almost at the same distance as the source. We did check other distances of the source from the first interface. Indeed, a distance of $0.30 \lambda$ was examined. As expected the image moves closer to the second interface at roughly $0.09 \lambda$. However, the distance of the image from the second interface is not at the same position as if our structure was isotropic with a refractive index equal exactly to -1 . This is due to the anisotropy of our structure.

One way to verify the good quality of the image compared to the source is to plot the average field intensity over a period at both the source and image planes. Figure 10 displays the normalized average field intensity versus the lateral direction for the cut structure. The full width at half maximum (FWHM) of the image beam is $0.35 \lambda$ and it is almost the same as the FWHM at the source. This result shows that the cut structure, which supports surface waves focuses in perfect way the object. Thus with a source that is not too close to the structure $(0.15 \lambda)$ we succeed in exciting the evanescent waves and achieving a better focus.

Apparently our structure with $0.10 a$ cut surface seems to be a good candidate for superlensing because we achieved a quite high transmission over a wide range of angles. Figure 11 presents the transmission versus the angle of incidence for different values of surface terminations. By trying different terminations we managed to optimize the transmission. For the complete structure, the transmission start at $80 \%$ for normal incidence, it gets high for a short range of angles then it drops to less than $20 \%$ for angles larger than 70 (deg). However, the structure with a surface termination of $0.10 a$ (see Fig. 11) shows the maximum transmission for all incident angles. Thus, the transmission is about $95 \%$ for angles up to 


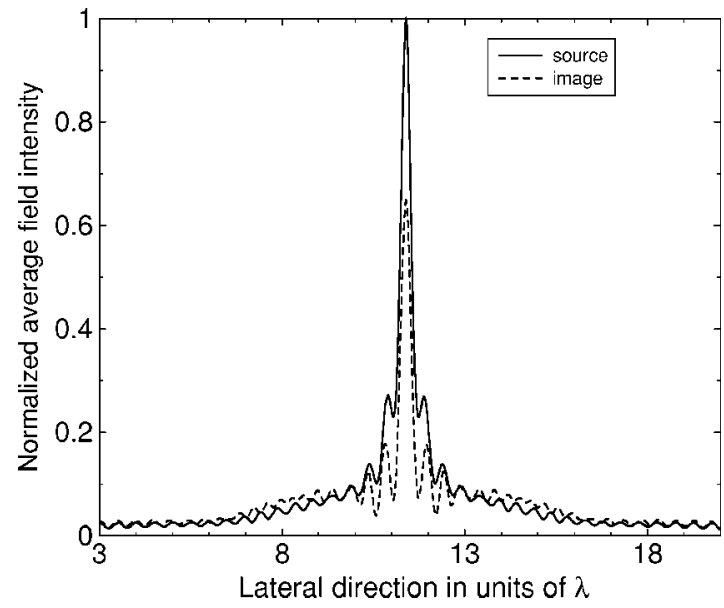

FIG. 10. The normalized average field intensity at the source (black curve) and image plane (dashed curve) vs the lateral direction.

40 (deg), and more than $80 \%$ for angles up to 65 (deg) and more than $50 \%$ for angles up to $78(\mathrm{deg})$. This result shows first how the transmission gets enhanced by the means of the excitation of the surface waves for a nonhomogeneous structure. Second, high transmission over a wide range of incident angles excludes the possibility of the funneling effect ${ }^{14,15}$ and accentuate the fact that the negative refraction in our structure is purely a result of LH behavior ${ }^{5}$ and not a preferred propagation direction.

In conclusion, we have systematically studied a LH structure based on a PC with a negative refractive index. Our experimental and theoretical results show the negative refraction as well as the superlensing phenomenon in this structure. Furthermore, surface waves at the interface be-

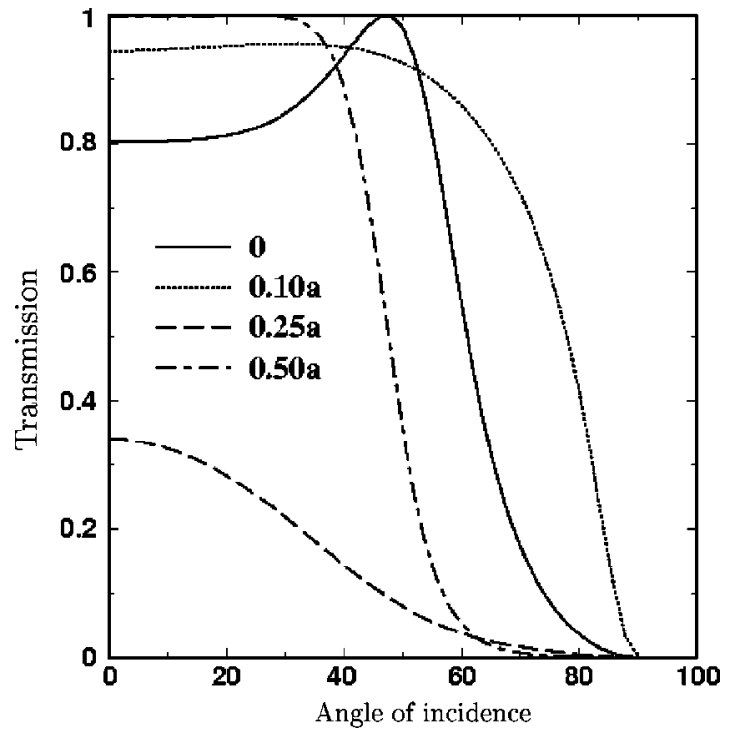

FIG. 11. Transmission vs the angle of incidence for different surface terminations.

tween air and the PC are excited within a specific termination of the surface allowing the reconstruction of the evanescent waves for better focus. The calculated average field intensity in the source plane as well as in the image plane shows almost the same full width at half maximum demonstrating the perfect image reproduced by this structure. Its high transmission along a wide range of angles makes it a good candidate for observing LH behavior in PCs.

This work was partially supported by Ames Laboratory (Contract No. N-7405-Eng-82), DARPA (Contract No. MDA972-01-2-0016), and EU-DALHM.
${ }^{1}$ V. G. Veselago, Sov. Phys. Usp. 10, 509 (1968).

${ }^{2}$ R. A. Shelby, D. R. Smith, and S. Schultz, Science 292, 779 (2001).

${ }^{3}$ E. Cubukcu, K. Aydin, E. Ozbay, S. Foteinopoulou, and C. M. Soukoulis, Nature (London) 423, 604 (2003).

${ }^{4}$ P. Markos and C. M. Soukoulis, Phys. Rev. B 65, 033401 (2001).

${ }^{5}$ S. Foteinopoulou and C. M. Soukoulis, Phys. Rev. B 67, 235107 (2003); cond-mat/0403542 (unpublished).

${ }^{6}$ M. Notomi, Phys. Rev. B 62, 10696 (2000).

${ }^{7}$ C. Luo, S. G. Johnson, J. D. Joannopoulos, and J. B. Pendry, Phys. Rev. B 65, 201104(R) (2002); 68, 045115 (2003).

${ }^{8}$ E. Cubukcu, K. Aydin, E. Ozbay, S. Foteinopoulou, and C. M. Soukoulis, Phys. Rev. Lett. 91, 207401 (2003).

${ }^{9}$ S. Foteinopoulou, E. N. Economou, and C. M. Soukoulis, Phys.

Rev. Lett. 90, 107402 (2003).

${ }^{10}$ J. B. Pendry, Phys. Rev. Lett. 85, 3966 (2000).
${ }^{11}$ R. Moussa and C. M. Soukoulis (unpublished).

${ }^{12} \mathbf{S}$ is the time averaged quantity of the spatial average of the Poynting vector within the unit cell.

${ }^{13}$ A. Taflove, Computational Electrodynamics-The Finite Difference Time-Domain Method (Artech House, Boston, 1995); K. S. Yee, IEEE Trans. Antennas Propag. 14, 302 (1966).

${ }^{14}$ Z. P. Liao, H. L. Wong, B. P. Yang, and Y. F. Yuan, Sci. Sin., Ser. A 27, 1063 (1984).

${ }^{15}$ Z.-Y. Li and L.-L. Lin, Phys. Rev. B 68, 245110 (2003).

${ }^{16}$ L.-S. Chen, C.-H. Kuo, and Z. Ye, Phys. Rev. E 69, 066612 (2004).

${ }^{17}$ P. Kramper, M. Agio, C. M. Soukoulis, A. Birner, F. Müller, R. B. Wehrspohn, U. Gösele, and V. Sandoghdar, Phys. Rev. Lett. 92, 113903 (2004).

${ }^{18}$ E. Moreno, F. J. Garcia-Vidal, and L. Martin-Moreno, Phys. Rev. B 69, 121402(R) (2004). 